\title{
Metodologia e validação de design estrutural baseado em desempenho para concepção de estrutura de torre eólica
}

\author{
Methodology and validation of performance-based \\ structural design applied to the conception of a lattice \\ wind tower
}

\begin{abstract}
Marina Ferreira Borges Universidade Federal de Minas Gerais Belo Horizonte - MG - Brasil

Lucas Figueiredo Grilo Universidade Federal de Minas Gerais Belo Horizonte - MG - Brasil

Ricardo Hallal Fakury Universidade Federal de Minas Gerais Belo Horizonte - MG - Brasil

Recebido em 25/03/16 Aceito em 12/07/16

\section{Marina Ferreira Borges \\ Lucas Figueiredo Grilo \\ Ricardo Hallal Fakury}

\section{Resumo}

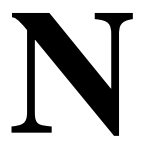

este trabalho é estudado o processo de projeto baseado em desempenho por meio do desenvolvimento de modelos paramétricos e algorítmicos, integrando análise estrutural ao design para a geração de estruturas geométricas complexas, com base em parâmetros de produção e montagem. Essa metodologia baseia-se no Modelo Performativo, em que a forma é gerada utilizando-se critérios de desempenho. Nessa abordagem o desenvolvimento de estruturas complexas só é possível pela reversão do processo de concepção da forma, cuja geração está atrelada aos parâmetros de geometria, material e carregamento. Assim, o engenheiro estrutural não participa apenas da fase de avaliação, mas também das fases iniciais do processo, criando um processo de pesquisa e produção de conhecimento comum entre os arquitetos e engenheiros. Para investigar essa abordagem, neste artigo é feita a concepção da estrutura de uma torre de energia eólica.

Palavras-chaves: Design estrutural. Arquitetura paramétrica e algorítmica. Análise estrutural. Modelo baseado em desempenho. Torre de energia eólica.

\section{Abstract}

This paper presents a study based on the performance design process by using parametric and algorithmic models development. This study integrates the structural analysis and the design to generate complex geometric structures, based on manufacture and assembly parameters. The used methodology is based on the Performative Model, in which the structure shape is generated using performance criteria. In this perspective, the complex structures development is only possible by reverting de shape conception process, in which its generation is linked to the geometric, material and load parameters. Therefore, the structural engineer not only participates in the evaluation phase, but also in the early stages, creating an exploration and production process of common knowledge among architects and engineers. For the purpose of investigating this approach, this work presents the design of a conceptual lattice wind tower.

Keywords: Structural design. Parametric and algorithm architecture. Structural analysis. Performative model. Lattice wind tower.
\end{abstract}




\section{Introdução}

No começo do século XXI houve uma mudança de metodologia na arquitetura com a evolução para a geração digital da forma, na qual o computador passou a ser utilizado para explorar todas as possibilidades inerentes ao processo em curso. Nessa nova maneira de se projetar, o arquiteto deixa de modelar as formas para articular uma lógica de geração das formas (TERZIDIS, 2011). É nesse contexto de mudança de paradigmas que se abriram as possibilidades de simulação da forma através de processos de otimização ou de geração, nos quais os arquitetos começaram a desenvolver seus projetos a partir do desempenho. A arquitetura paramétrica e algorítmica permite que os modelos sejam concebidos com base em relações paramétricas e através de scripts, e que sejam colocados dados matemáticos como base de geração da forma.

Dessa maneira, neste artigo procurou-se de maneira experimental desenvolver um projeto baseado em desempenho, aplicado ao projeto estrutural através de uma reversão da maneira de se projetar a forma. Shea, Aish e Gourtovaia (2005) dizem que "a próxima fase do design digital encontra-se em considerar o computador como um parceiro de colaboração no processo de design que é capaz de gerar novas ideias de design e estimular soluções das mais difíceis tarefas". Para isso, foram utilizadas as ferramentas digitais emergentes, nas quais se pode combinar o desenvolvimento da geometria com a análise estrutural, explorando os benefícios da reaproximação dos trabalhos do arquiteto e do engenheiro de estruturas, através da redefinição das posturas desses dois profissionais.

Nesse contexto, este trabalho apresenta um estudo sobre uma torre eólica de estrutura treliçada, com dimensões aproximadas de $140 \mathrm{~m}$ de altura e $30 \mathrm{~m}$ de diâmetro de base, para instalação de um aerogerador de 3 MW. Foi desenvolvido um algoritmo para definir a geometria de uma casca equivalente à estrutura da torre, com os softwares Rhinoceros, Grasshoper e Scan\&Solve, e posteriormente determinar a espessura necessária em cada trecho da casca devido aos esforços solicitantes. De acordo com a espessura requerida em cada trecho, foi feita uma equivalência entre a casca obtida e os perfis tubulares com os quais se pretendia utilizar na estrutura, e, por fim, definida a geometria final da treliça. Para a validação do procedimento, foi feita a verificação estrutural da treliça encontrada utilizando-se o software SAP 2000 .

\section{Design do design}

\section{Form-finding}

$\mathrm{O}$ processo de form-finding vem sendo utilizado na arquitetura desde a segunda metade do século $\mathrm{XX}$, não tendo estado em um primeiro momento associado ao desenvolvimento de tecnologias digitais. Segundo Oxman (2010), no form-finding as formas emergem de uma integração entre o material e o carregamento, através de um ou mais critérios. Quando levado ao extremo, a forma é exclusivamente guiada pela função, sendo uma expressão pura de aplicação da engenharia e de métodos de otimização. Vladimir Shukhov, Frei Otto e Buckminster Fuller são exemplos de pioneirismo do uso desse tipo de metodologia científica, em que a pesquisa e a tecnologia tornaram-se ferramentas de projeto. Buckminster Fuller ficou conhecido por desenvolver cúpulas geodésicas com estruturas triangulares, buscando o máximo de volume com o mínimo de superfície, de tal maneira que o peso total também fosse minimizado. Vladimir Shuckhov foi pioneiro em estruturas do tipo gridshell, como, por exemplo, a estrutura hiperbólica da torre de Shabolovka.

Frei Otto (ARCHDAILY, 2012) também desenvolveu um processo de form-finding para encontrar superfícies mínimas através de seus experimentos com bolhas de sabão, que teve aplicação em estruturas tensionadas (Figura 1). Ele aplicou esse processo, entre outras obras, no German Pavilion para a Expo'67 (Figura 2), que, embora aparente ser uma estrutura estética e tecnicamente sofisticada, tem uma concepção conceitualmente simples, com poucos componentes, objetivando facilitar a montagem e a adaptação a diferentes tipos de terrenos.

Além do processo de form-finding, Frei Otto também foi defensor da pesquisa interdisciplinar na arquitetura e trabalhou de maneira colaborativa com diversos engenheiros, como Ove Arup e Félix Candela. Suas maquetes físicas foram importantes tentativas de se encontrarem superfícies mínimas, mas atualmente o uso de ferramentas digitais emergentes levou as ideias de modelos experimentais desenvolvidas por ele a um novo patamar de avaliação e síntese da forma. 
Figura 1 - Experimento com bolhas de sabão para encontrar superfícies mínimas

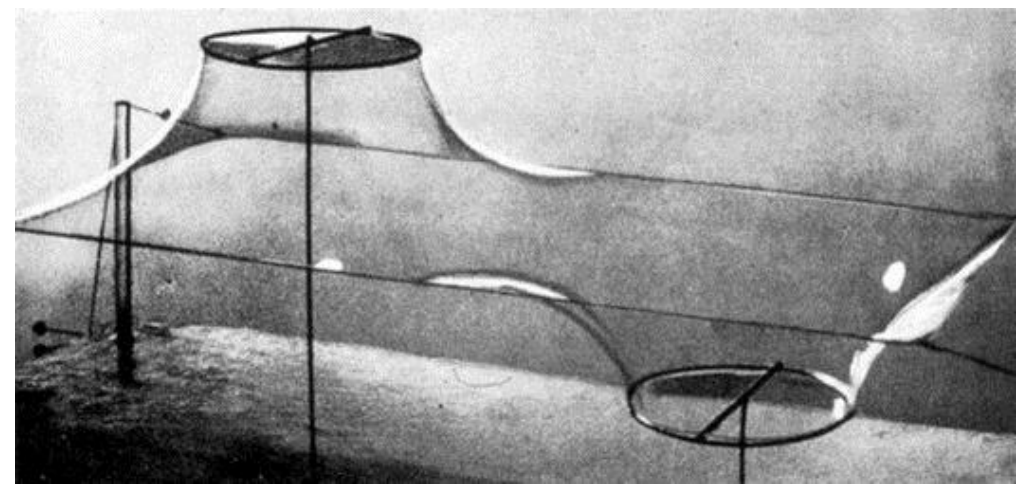

Fonte: Archdaily (2012).

\section{Figura 2 - German Pavilion para a Expo'67}

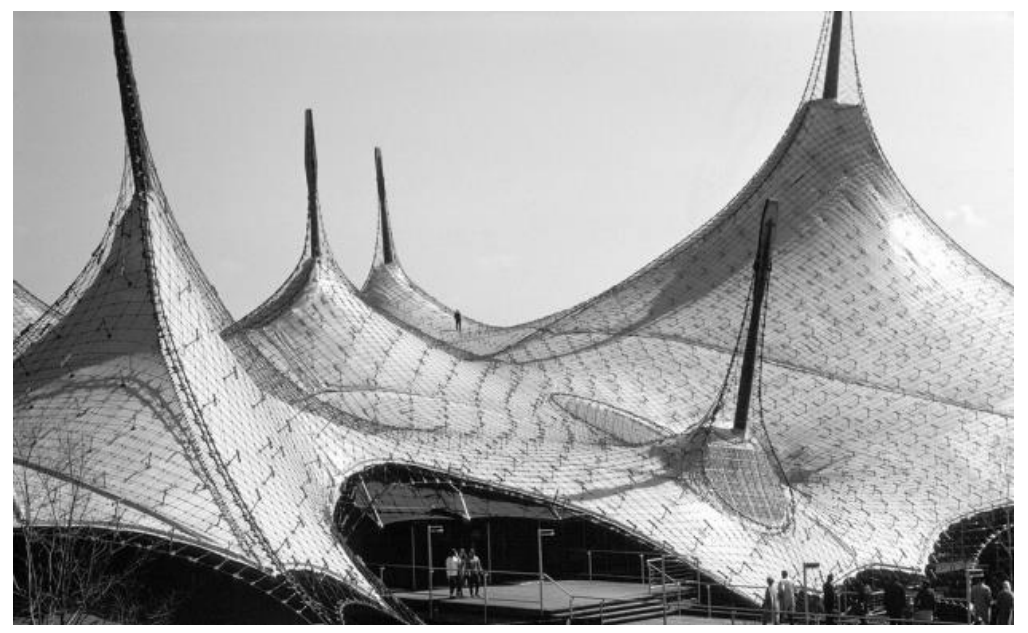

Fonte: Archdaily (2012).

\section{Projeto baseado em desempenho}

Os processos de form-finding criam inúmeras possibilidades de formas heterogêneas quando trabalhados com softwares paramétricos e algorítmicos. O projeto baseado em desempenho surge como um instrumento que contribui para a concepção de projetos inovadores, com a integração de mecanismos de simulação e geração, exigindo dos profissionais posturas colaborativas no processo de projeto. Kolarevic (2005) afirma que questões qualitativas e quantitativas de desempenho devem ser colocadas como princípios tecnológicos que guiarão novas abordagens de projeto. Oxman (2006) cita três componentes essenciais para o sistema de design digital:

(a) modelo geométrico capaz de gerar e transformar a forma através de inputs dados pelos processos de avaliação;

(b) processos de avaliação integrados com o modelo geométrico; e (c) o designer como um moderador do processo, de maneira interativa e iterativa.

Para o projeto baseado em desempenho, Oxman (2006) ressalta a importância do desenvolvimento de modelos paramétricos e algorítmicos. O modelo paramétrico cria relações de interdependência entre as partes do projeto e possibilita que elas sejam facilmente transformadas à medida que forem ocorrendo as alterações exigidas pelos critérios estabelecidos. Suas principais características são a adaptabilidade, a mudança, a continuidade e a conectividade. Com isso, as técnicas de otimização podem se integrar ao processo de modificação da forma, de forma a permitir que o designer desenvolva métodos automatizados e semiautomatizados de geração e avaliação. Oxman (2006) propôs um modelo de critérios de desempenho na avaliação do projeto que coloca o projetista simbolicamente no centro e em interação com os demais componentes (Figura 3). 
Figura 3 - Generic schema representado por representação (R), avaliação (E), performance $(P)$ e geração (G)

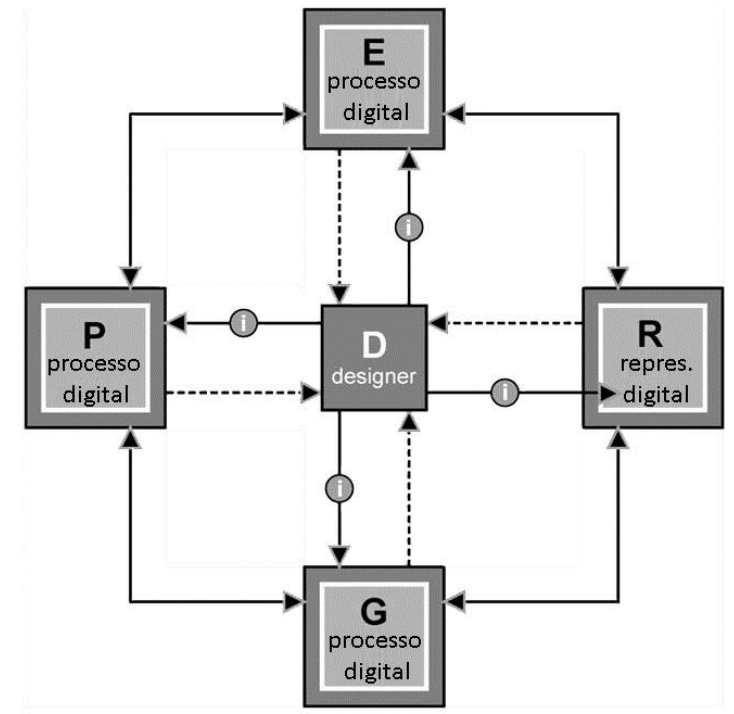

Fonte: adaptado de Oxman (2006).

O paradigma de projeto baseado em desempenho foca menos em questões formais e estéticas, e mais no material e na inteligência do design. A forma final não é tão relevante, sendo a lógica que a define o mais importante. De acordo com Grobman (2012), um novo tipo de arquitetura baseada em dados e desempenho está gradualmente sendo desenvolvido, em contraste com as arquiteturas focadas em tipologia.

As possibilidades de implementação de processos generativos têm crescido significativamente. No princípio dos anos 2000 apenas escritórios de elite, com condições para ter equipes de programadores, como $o$ escritório de arquitetura inglês Foster+Partners, tinham recursos para o desenvolvimento desses processos. Um exemplo de aplicação foi a cobertura de vidro do British Museum, em Londres, concebida em parceria com o escritório de engenharia Buro Happold (Figura 4).

Atualmente, os softwares paramétricos, como o Grasshoper, têm tornado essa metodologia cada vez mais conhecida. $\mathrm{O}$ projeto baseado em desempenho reflete as mudanças na própria profissão de arquiteto e na filosofia, na qual os profissionais parceiros, principalmente o engenheiro estrutural, têm fundamental importância na concepção da forma.

Em uma abordagem contemporânea para o desenvolvimento do projeto de estruturas, a sequência de decisões de projeto parte da estrutura e do material, para posteriormente ser definida a forma. Essa abordagem foi denominada por Oxman e Oxman (2010) como Novo
Estruturalismo. Tradicionalmente, a forma é concebida pelo arquiteto e posteriormente analisada e otimizada pelo engenheiro de estruturas. Para Oxman e Oxman (2010), a engenharia de vanguarda aproxima a otimização e a geração dos projetos estruturais com os conceitos de arquitetura, revertendo a maneira de pensar forma, força, estrutura e desempenho. O Novo Estruturalismo é a integração de design, arquitetura, engenharia estrutural e tecnologias digitais. $\mathrm{O}$ estudo de geometrias complexas e as possibilidades de projeto arquitetônico através de algoritmos vêm redefinindo as possibilidades do projeto estrutural. Os softwares paramétricos foram extremamente importantes no desenvolvimento desses conceitos. Eles são um meio de produzir design generativo, estrutural e iterativo, através de processos colaborativos entre arquitetos e engenheiros.

\section{Projeto para a fabricação}

A primeira geração de arquitetos que desenvolveu projetos baseados na geração digital da forma experimentou uma euforia devido à exploração formal que as ferramentas possibilitavam. Superado esse momento inicial, começaram-se a trabalhar as possibilidades de explorar a lógica do material e dos métodos de montagem e produção. A integração de projeto, fabricação e montagem embutidos na lógica do design fez com que as etapas de concepção fossem racionalizadas e que o trabalho se tornasse multidisciplinar e colaborativo. 
Figura 4 - Simulações computacionais de subdivisão da cobertura de vidro do British Museum
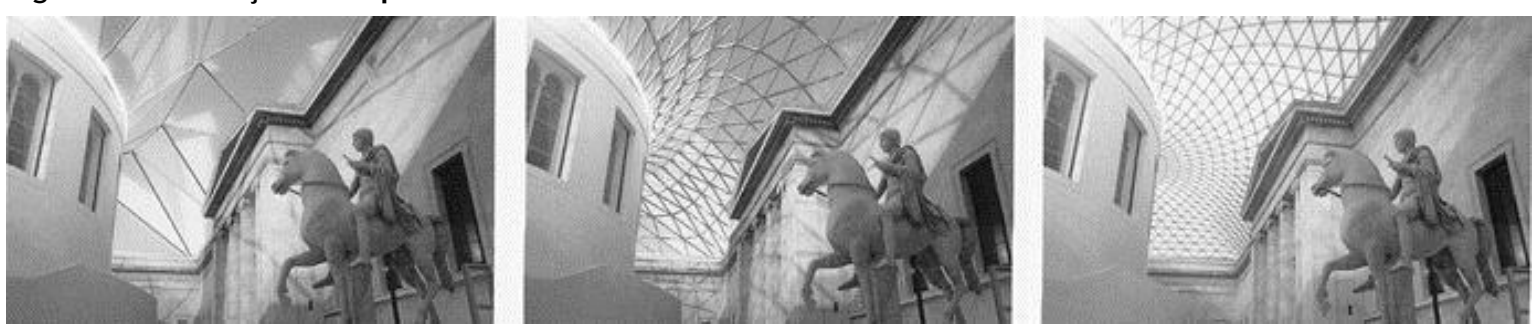

Fonte: Burry e Burry (2010).

As possibilidades do design se tornaram ainda mais amplas através das técnicas de fabricação digital. O pensamento voltado para a modulação da produção em massa foi substituído pela lógica da customização em massa, fazendo com que as técnicas de fabricação se tornassem uma extensão do pensamento do design. Tecnicamente, design e fabricação convergiram para uma linguagem única através de um código digital, tendo um único meio de comunicação entre o desenho do arquiteto e o processo de fabricação. No Brasil os processos de integração de design e fabricação digital ainda são incipientes, com algumas experiências feitas apenas em ambiente acadêmico. Portanto, mesmo os projetos desenvolvidos dentro de uma lógica de geração da forma devem ser submetidos à produção da indústria tradicional.

Este artigo procurou, de maneira experimental, desenvolver uma forma através de ferramentas digitais em que o desenvolvimento do algoritmo associasse estética, produção e montagem. Em um primeiro momento, algumas questões foram colocadas como primordiais para a definição do estado da arte do assunto no Brasil: seria possível dentro da cultura de especialização do trabalho na construção civil que os trabalhos de arquitetos e engenheiros estruturais pudessem convergir a partir de práticas digitais emergentes? Seriam as ferramentas digitais disponíveis suficientes para o desenvolvimento de um projeto baseado em desempenho, ou esse tipo de metodologia ainda é inacessível fora da elite da arquitetura? Como seria o desenvolvimento desse modelo com os recursos disponíveis no Brasil para um projeto de produção em massa com o uso da indústria tradicional?

Para isso, através do desenvolvimento de um projeto experimental baseado em desempenho, foram exploradas as ferramentas digitais emergentes, com as quais se podem combinar a definição da geometria com a análise estrutural. Além desse enfoque, foi explorada uma quebra de paradigmas da especialização dos trabalhos de engenheiros e arquitetos. Com o desenvolvimento do projeto foi proposta uma integração do engenheiro de estruturas e da indústria com o arquiteto, para conceber a forma de maneira otimizada.

Como objeto de aplicação, neste artigo foi efetuado o desenvolvimento do projeto de uma torre de energia eólica composta de perfis tubulares laminados de aço de seção circular (tubos circulares) fabricados pela Vallourec do Brasil (único fabricante nacional desse produto) com aço VMB 350, tendo em vista a relevância de se gerar energia sustentável no Brasil. Para tanto, a torre requer uma solução otimizada do ponto de vista estrutural, mas também necessita de uma solução estética que não gere poluição visual, pois muitas vezes ela é instalada em locais de grande afluxo de pessoas e até em locais turísticos. O desenvolvimento do design da torre é de suma importância para o trabalho e é o principal fator que demanda a participação do arquiteto no processo de concepção da forma da estrutura. Como as questões estruturais, de produção e montagem estão intrinsecamente relacionadas à forma, a proposta é que seja utilizado um processo colaborativo de design, com o uso de ferramentas digitais. O objetivo é que se chegue a uma forma, concebida através da combinação de técnicas generativas e de análise estrutural, que tenha transparência e leveza estética e que também leve a um peso aceitável da estrutura (um peso muito elevado pode significar a inviabilidade do projeto por questões econômicas).

\section{Primeiro passo: modelagem paramétrica}

\section{Parâmetros geométricos}

A torre eólica a ser desenvolvida tem como proposta captar as melhores correntes de vento, que ocorrem a partir de determinadas alturas em relação ao solo, nas quais os aerogeradores de maior potência são eficientes. Para isso foi estipulada para ela uma altura de $140 \mathrm{~m}$, valor equivalente ao das maiores torres no mundo presentemente. Para a definição dos parâmetros de geometria e carregamento do projeto, a escolha da turbina foi fundamental. Assim, foi escolhida uma 
turbina de $3 \mathrm{MW}$, potência situada na faixa superior das turbinas em operação atualmente, com três pás de aproximadamente $60 \mathrm{~m}$ de comprimento, tendo como referência a GWT3.0MW (ANDREA, 2014). Em consequência desse comprimento, a torre precisou ter duas partes, uma superior, que ladeia as pás, com $60 \mathrm{~m}$ de altura, e uma inferior, com $80 \mathrm{~m}$. Para o dimensionamento da parte inferior da torre, buscaram-se casos relatados na literatura, como em Engström et al. (2010), no qual o diâmetro da base adotado para uma torre eólica treliçada de $150 \mathrm{~m}$ de altura com turbina de $3 \mathrm{MW}$ foi de $30 \mathrm{~m}$. Portanto, esse também foi o diâmetro da base adotado para o projeto.

Na parte superior a seção transversal da torre deve ter um diâmetro máximo, que é função do projeto do aerogerador e da flecha máxima que pode ocorrer nas extremidades das pás. Em resumo, deve-se assegurar que para todas as condições de carregamento as pás não colidam com a estrutura da torre, com o aerogerador girando em $360^{\circ}$. Desse modo, optou-se por adotar para essa parte um diâmetro igual a $5 \mathrm{~m}$, com base nas investigações de Andrea (2014). Com as definições apresentadas, chegou-se aos parâmetros geométricos da torre mostrados na Figura 5.

Como o equipamento gira em torno da torre para a captura do vento em qualquer direção, considerouse conveniente que a estrutura da torre fosse projetada com um eixo axial de simetria ao longo de toda a sua altura.

\section{Tensão resistente usada na geração da forma}

Para a tensão resistente de cálculo foi assumido o seguinte valor (Eq. 1):
$\sigma_{R d}=\frac{\chi f_{y}}{\gamma_{a 1}}$

Eq. 1

Onde:

$f_{y}$ é a resistência ao escoamento do aço, igual a 350 MPa para o aço VMB 350;

$\gamma_{a 1}$ o coeficiente de ponderação da resistência do aço, igual a 1,10; e

$\chi \mathrm{o}$ fator de redução associado à força de compressão resistente.

Essa expressão foi utilizada considerando que todas as barras, dependendo do sentido do carregamento horizontal atuante, estarão submetidas tanto à compressão axial quanto à tração axial, sendo a compressão a condição mais desfavorável. Simplificadamente, no modelo de geração da forma adotou-se para $\chi$ o valor de 0,80 , correspondente a um índice de esbeltez reduzido da ordem de 0,90, ou a um índice de esbeltez igual a 60. Esse valor médio foi selecionado devido ao fato de que, a princípio, a esbeltez das barras não ultrapassaria esse valor. A flambagem local das barras não foi considerada, uma vez que os perfis laminados tubulares circulares produzidos pela Vallourec não são suscetíveis a esse modo de falha, por terem valores reduzidos da razão entre diâmetro e espessura. Todos os parâmetros de cálculo citados têm por base a norma brasileira NBR 16239 (ABNT, 2013). Aplicando os valores de fy, $\chi$ e $\gamma$ al à Equação 1, chega-se a um valor de $\sigma \mathrm{Rd}$ igual a 254,54 $\mathrm{MPa}$, indicando o valor máximo que a tensão normal de compressão pode alcançar.

\section{Figura 5 - Parâmetros geométricos para desenvolvimento da torre eólica}

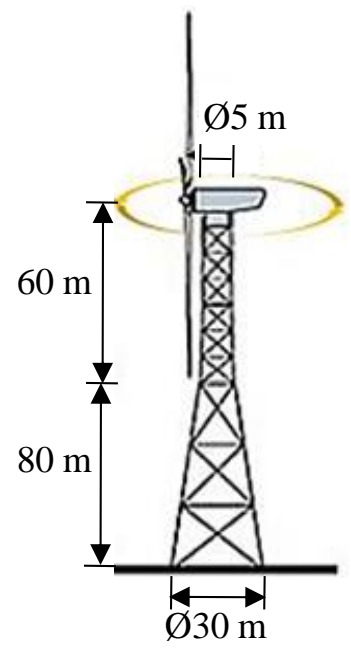

98 Borges, M. F.; Grilo, L. F.; Fakury, R. H. 


\section{Forças e momentos solicitantes de cálculo}

Neste trabalho não serão feitas maiores considerações a respeito das forças e momentos atuantes usados na análise estrutural, mas apenas fornecidos seus valores, tomados com base em Andrea (2014) e conservadoramente selecionados entre os mais altos de cada uma das combinações últimas de ações aplicáveis. Assim, foram assumidos a força $\mathrm{Fz}$, igual a $2.780 \mathrm{kN}$, devida ao peso próprio da estrutura e do equipamento (incluindo o aerogerador e as pás), a força FHres, igual a $955 \mathrm{kN}$, causada pela ação do vento na estrutura e no equipamento, e o momento Mres, igual a 14.115 kN.m, referente à excentricidade somada do posicionamento do equipamento e da ação de vento nesse equipamento. Essas ações foram aplicadas simplificadamente no topo da torre, como se vê na Figura 6, e permitiram chegar à tensão solicitante de cálculo.

\section{Segundo passo: desenvolvimento do algoritmo}

Para a análise estrutural foi escolhido o plugin Scan\&Solve (SCAN \& SOLVE, 2014), que exige dos usuários pouco conhecimento prévio em sistemas estruturais ou malha de elementos finitos. O modelo gerado é uma simplificação de uma malha, com o uso da tecnologia meshfree, patenteada pela empresa Intact Solutions. Segundo o fornecedor, o modelo é vantajoso por criar uma separação entre as representações geométrica e física do objeto, combinando-as quando necessário, sem dispender muito tempo com conversão e correção de erros da malha, e preservando a maioria das vantagens de método clássico de análise pelo MEF. Sendo assim, o Scan\&Solve apresenta-se como um software de análise estrutural viável para que os arquitetos o utilizem na concepção e pré-dimensionamento, além de estar integrado com a modelagem geométrica no Rhinoceros através do Grasshopper.

A primeira etapa de desenvolvimento do projeto consistiu em gerar uma geometria inicial em forma de casca por meio do Grasshoper (Figura 7). A solução tradicional da forma sugerida apenas pelo ponto de vista da engenharia indicava a combinação de um cilindro na parte superior com um tronco de cone na base. Para se encontrar uma forma que atendesse a fatores estéticos, foram utilizados os princípios da Gestalt, principalmente os que sugerem a continuidade e o arredondamento da forma, fazendo com que os olhos percorram de maneira suave a configuração do objeto (GOMES FILHO, 2000). A modelagem da casca, utilizando esses princípios, só foi possível com o uso de ferramenta nurbs, por se aproximar de um traço mais fluido, como no desenho à mão, permitindo o desenvolvimento de formas cada vez mais complexas e esteticamente leves.

O objetivo de primeiramente gerar uma casca foi definir uma forma estética inicial, e fazer a análise e dimensionamento prévios dessa casca com o programa Scan\&Solve, envolvendo a determinação das espessuras necessárias, em vários níveis, para que a tensão solicitante não ultrapassasse o valor da tensão resistente de cálculo, considerada como igual a 254,54 $\mathrm{MPa}$. Como critério de falha foi utilizada a formulação de von Mises. Alterando a ordem tradicional do processo de análise estrutural, a estrutura gerada foi construída de maneira otimizada, utilizando-se as tensões máximas como parâmetro para o desenvolvimento da estrutura com o menor número possível de nós. O processo teve como objetivo a geração da forma otimizada, com a análise estrutural passando a ter a função de geração, e não somente de validação da forma (Figura 8).

\section{Figura 6 - Forças aplicadas utilizadas para desenvolvimento da estrutura}

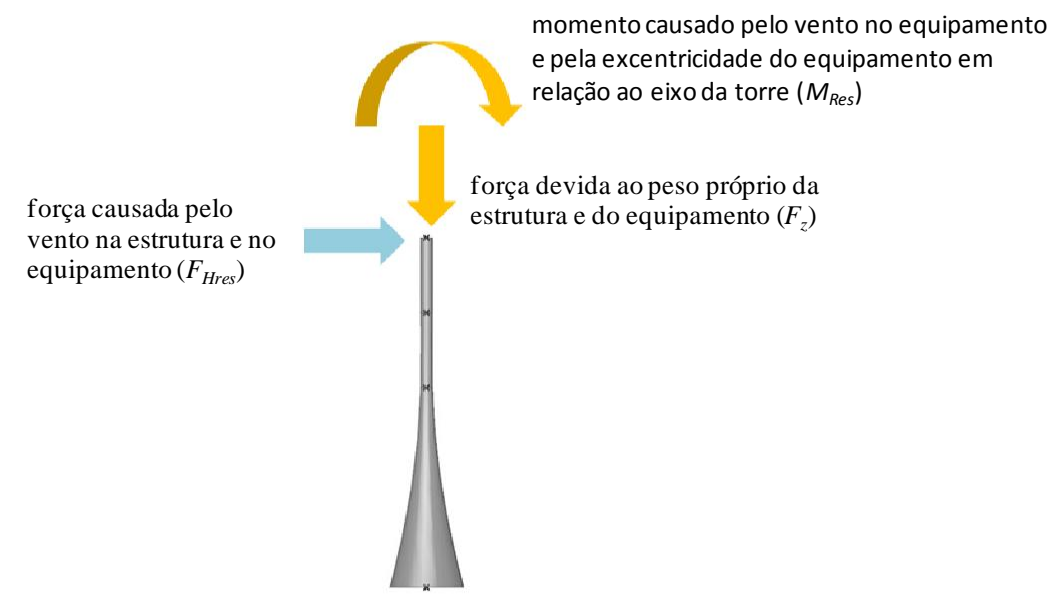


Figura 7 - Parâmetros de dimensionamento da casca em Rhinoceros e Grasshoper

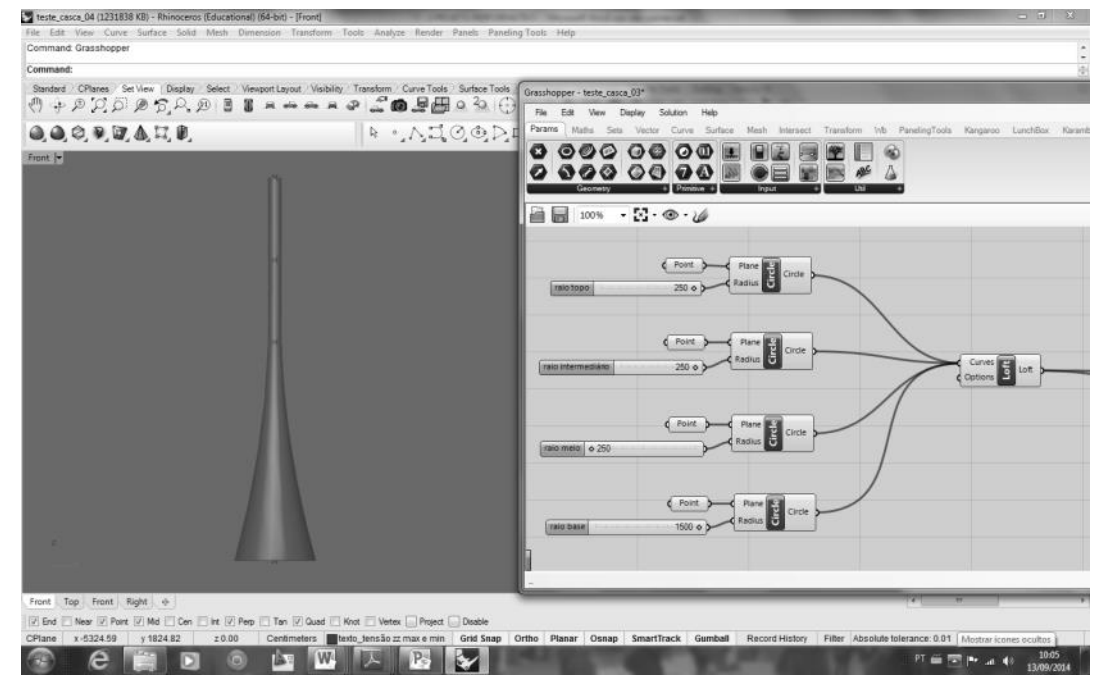

Figura 8 - Análise estrutural para geração da forma

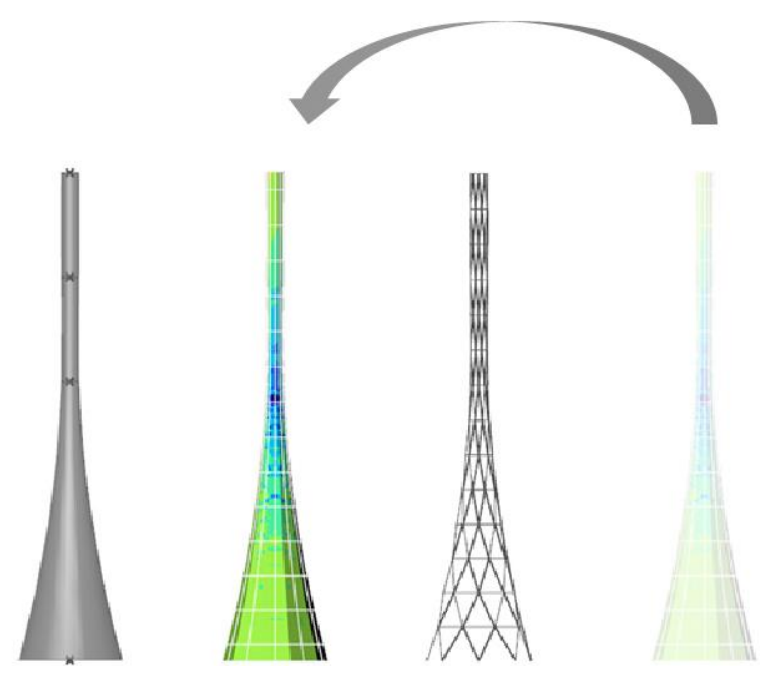

Para encontrar as espessuras máximas equivalentes em cada módulo da casca foi necessário adotar para a análise uma espessura de referência, tomada com valor igual a $10 \mathrm{~cm}$. Através da tensão máxima solicitante de cada módulo $(\sigma \mathrm{Sd})$ foi obtida uma espessura equivalente para o desenvolvimento da treliça, dada por (em centímetros) (Eq. 2):

$t=\frac{\sigma}{f_{y d}} \times 10$

Eq. 2

Com esse procedimento foi possível encontrar a área do anel em cada módulo e converter essa área em uma quantidade de tubos equivalente. Após a obtenção das tensões máximas em MPa em cada módulo, aplicando-se a Equação (2), foram encontradas as espessuras equivalentes mostradas na Figura 9.

A etapa seguinte de desenvolvimento do projeto da torre consistiu em analisar os dados de espessura da casca e relacioná-los com a quantidade de tubos equivalente em cada modulo da torre, que deveria se situar entre 4 e 12 . De acordo com a análise das tensões, a área inferior do anel em cada módulo serviu como referência para a área total das seções transversais necessária dos tubos (Figura 10). Contudo, fazendo essa equivalência, chegou-se a alguns módulos com quantidade de tubos superiores ao valor desejado. Por isso, através do Grasshoper, foi desenvolvido um algoritmo para o desenvolvimento da treliça composta dos perfis tubulares disponíveis no catálogo comercial de seções tubulares circulares da Vallourec Tubos do Brasil (2014), estabelecendo a relação da espessura da casca com a quantidade de perfis necessária para compor a forma final da torre, objetivando que cada módulo tivesse a quantidade de tubos dentro do intervalo desejado (Figura 10).

100 Borges, M. F.; Grilo, L. F.; Fakury, R. H. 


\section{Figura 9 - Relação entre tensão máxima e espessura equivalente em cada módulo}

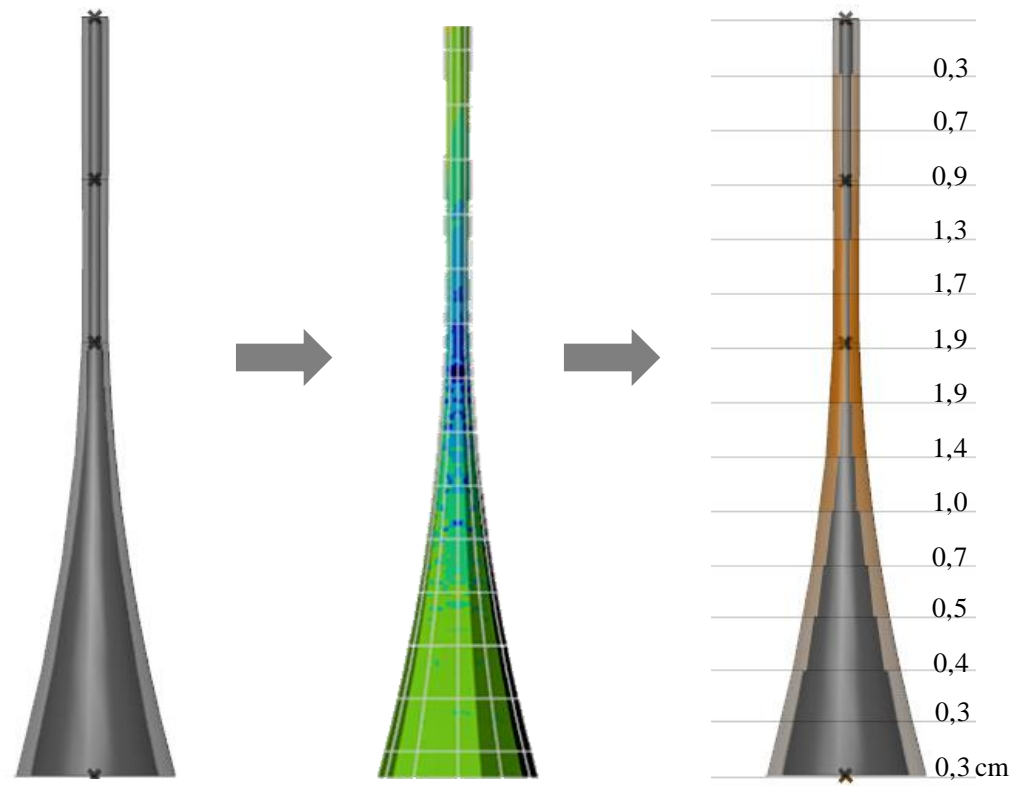

Figura 10 - Relação da espessura da casca com o desenvolvimento da estrutura

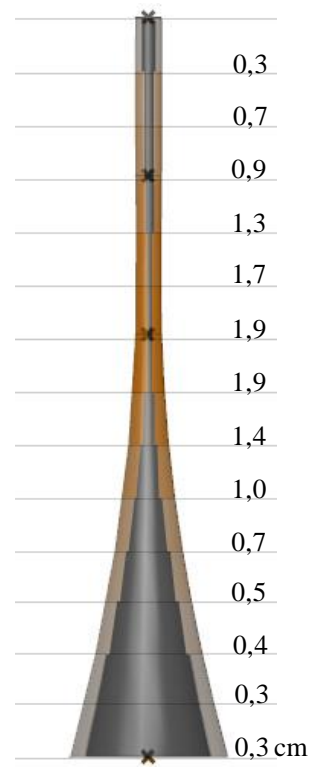

O cálculo da quantidade de tubos, $\mathrm{N}$, foi feito de acordo com a seguinte expressão (Eq. 3):

$$
N=\frac{A_{c}}{\sum A_{t}}
$$$$
\text { Eq. } 3
$$

onde Ac é a área da seção de cada módulo da casca e At é a área da seção transversal de cada tubo. Com essas definições foi possível o desenvolvimento do algoritmo gerador da treliça através do Grasshoper, feito de acordo com a Figura 11.

A programação em Visual Basic foi necessária para se obter de maneira automatizada a menor

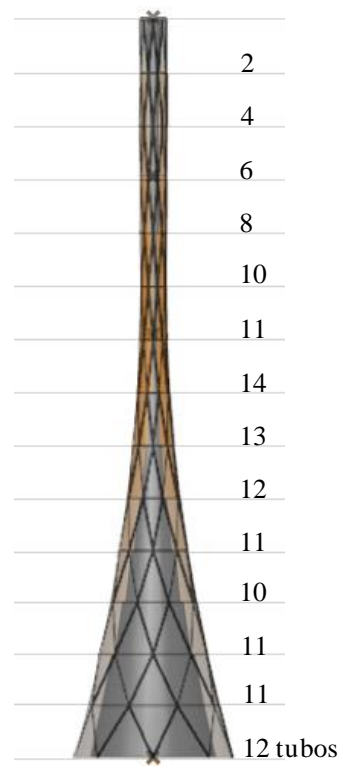

quantidade possível de tubos, determinada a partir das espessuras predeterminadas. Estabeleceu-se que a quantidade mínima seria de 4 tubos por módulo, e a quantidade máxima de 12 tubos, de tal maneira que as barras não tivessem inclinações menores do que $30^{\circ}$ em relação à horizontal. As tabelas dos tubos selecionados se conectaram diretamente ao código VB como parâmetro de entrada para as definições de condicionais. Além das tabelas, também foram definidos como parâmetros de entrada a espessura da casca e o raio externo da seção. Como se vê na Figura 12, os valores encontrados são classificados de forma booleana em adequados e não adequados. 
Figura 11 - Diagrama do esquema de montagem do algoritmo para estrutura

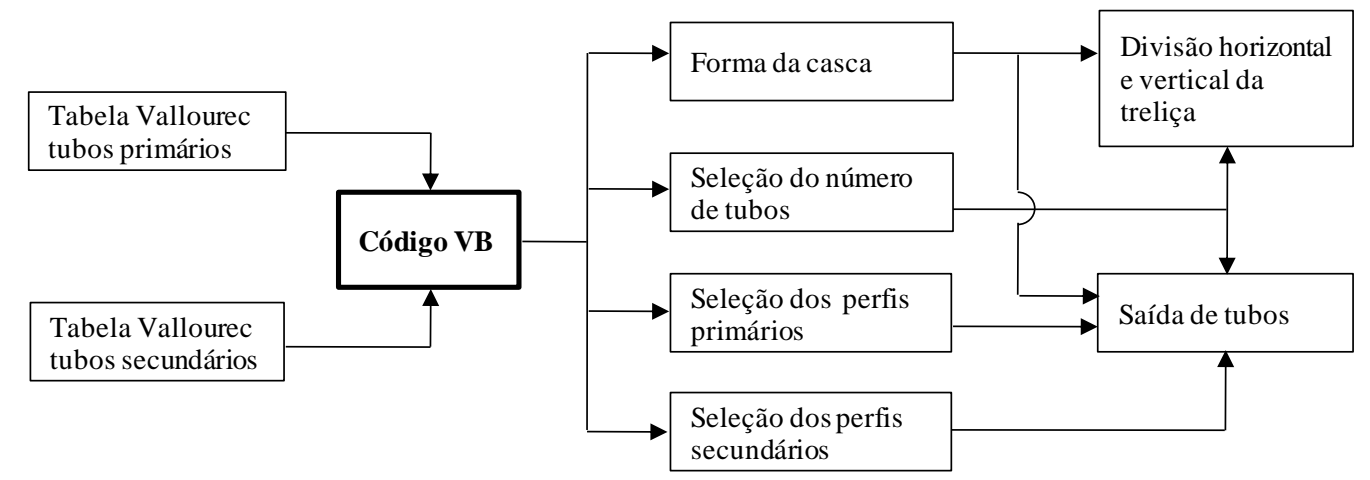

Figura 12 - Diagrama condicional para geração geométrica de tubos

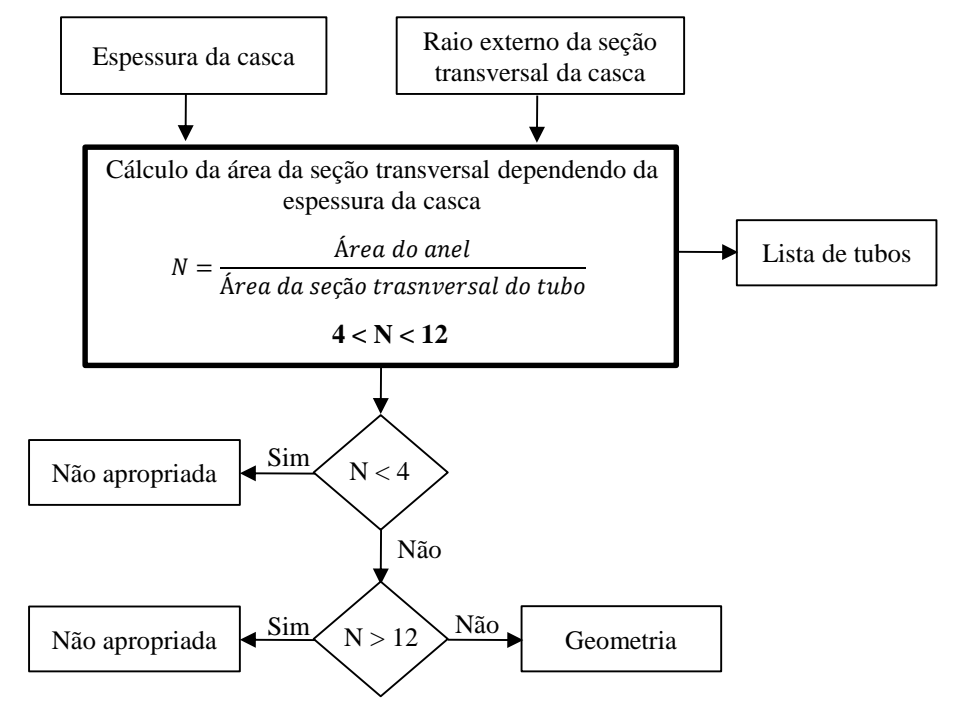

O parâmetro de saída dos tubos está diretamente conectado com os parâmetros de divisão da modulação e com os diâmetros dos tubos comerciais definidos através da condicional do código VB. O algorítimo implementado está apresentado a seguir, no qual é selecionada a menor seção transversal dos tubos necessária para que o seu somatório equivalha à seção transversal da casca, tendo como condicional a quantidade de tubos estar dentro do intervalo desejado (entre $4 \mathrm{e}$ 12):

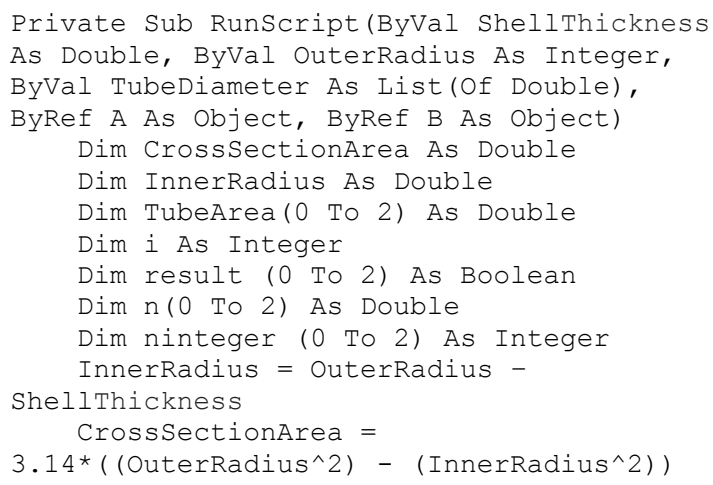

For $i=0$ To 2

TubeArea $(i)=3.14$ *

((TubeDiameter(i) / 2) ^ 2)

$\mathrm{n}(\mathrm{i})=$ CrosssectionArea /

TubeArea (i)

ninteger $(i)=$ math. Ceiling (n(i))

If ninteger(i) < 4 Then

result (i) $=$ False

print("valor não adequado")

ElseIf ninteger(i) > 12 Then

result (i) $=$ False

print("not appropriate value ")

Else

result (i) = True

print ("appropriate value")

End If

Next

$A=$ ninteger

$\mathrm{B}=$ result

End Sub

Fazendo as combinações possíveis com quantidades de tubos de 4 a 12 por seção transversal da torre (Figura 13), foram obtidas diversas opções de configuração para a treliça. Em seguida, foram estabelecidos alguns critérios para se encontrar a melhor solução, considerando fatores quantitativos e qualitativos. 
Para a definição dos perfis, foi utilizado o catálogo da Vallourec Tubos do Brasil (2014), segundo o qual o maior perfil disponível tem diâmetro de 355,6 mm. Contudo, com esse perfil alguns módulos tiveram uma quantidade de barras na seção horizontal superior a 12, o que não atendeu à condicional estabelecida. Para solucionar o problema, foi considerada a possibilidade de se usarem barras compostas formadas por 2 ou 3 tubos, dispostos concentricamente entre si, no sistema tubo dentro de tubo (Figura 14), para se obter a área necessária na seção horizontal da torre, conforme estudo de Grilo (2015). Como essa possibilidade envolve custos adicionais, definiu-se como critério evitá-la ao máximo, e na hipótese de ser necessária, procurar sempre que possível usar barras compostas com apenas 2 tubos.

Dessa maneira foi desenvolvido o processo paramétrico integrado com o algoritmo e com os parâmetros de tensão obtidos através da análise estrutural. A partir desse modelo puderam ser geradas inúmeras possibilidades a partir dos parâmetros estabelecidos (Figura 15).

Figura 13 - Distribuição de tubos na seção transversal da torre (caso de 8 tubos por seção transversal)

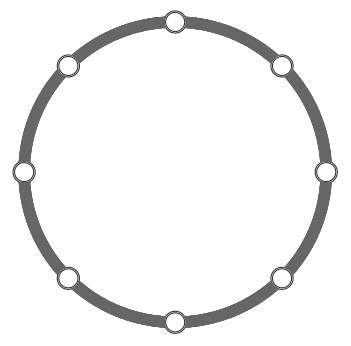

Figura 14 - Relação do catálogo comercial com o desenvolvimento da estrutura

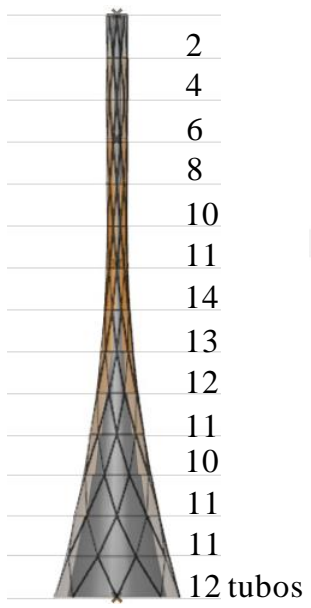

Catálogo comercial de perfis tubulares circulares da Vallourec

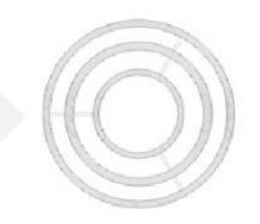

Combinação de 3 tubos: $\varnothing 355,6, \varnothing 273$ e $\varnothing 168,3 \mathrm{~mm}$

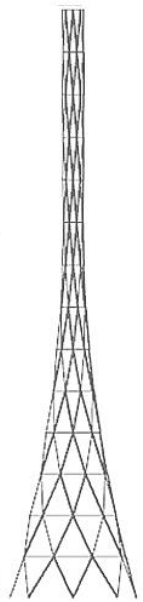

Mesmo número de tubos em cada módulo

Figura 15 - Estrutura do processo paramétrico e algorítmico

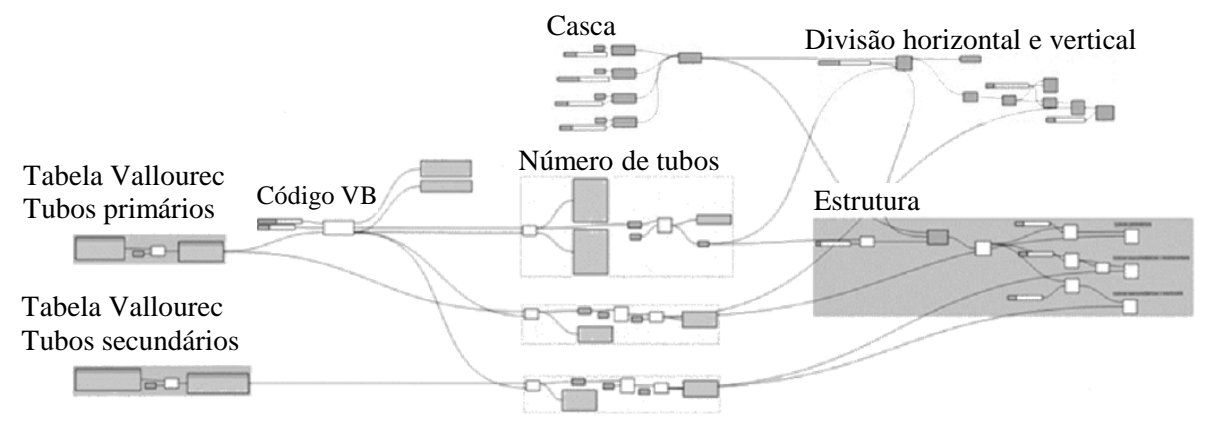


Para verificar comparativamente as combinações de tubos, na Tabela 1 foram colocados os demais critérios considerados determinantes para a decisão. A estrutura mais eficiente seria aquela que não necessitasse de barras compostas de 3 tubos, tivesse as menores variações de perfis ao longo da torre e tivesse a menor quantidade de módulos sem combinação de perfis.

Com esses critérios analisados, os melhores resultados obtidos foram para a torre com 8 perfis e para a combinação de 8 e 4 perfis, sendo necessária uma transição em determinada elevação. Entretanto, esteticamente a preferência seria pela continuidade da mesma quantidade de perfis, com o menor número de tubos, tendo como referência a regra de continuidade da forma proposta pela Gestalt (GOMES FILHO, 2000). Dessa maneira, a torre com 8 tubos obteve o melhor resultado, embora não tenha muitos módulos com o perfil de diâmetro de $355,6 \mathrm{~mm}$.

Sendo assim, a melhor geometria encontrada com essa metodologia foi a com 8 tubos em todos os 14 módulos, tendo os perfis primários o diâmetro de $355,6 \mathrm{~mm}$, e os secundários, de 168,3 mm. Essa geometria manteve todos os módulos com o mesmo aspecto estético, o que garantiu uma unidade visual à estrutura. Além desse fator, a utilização de um número constante de tubos na seção horizontal da torre tornou desnecessárias transições, as quais promoveriam ligações mais complexas.
Segundo Celani (2011), um sistema generativo pode ser utilizado visando à otimização (convergência) ou à variedade (divergência) por meio da geração de múltiplas alternativas. Esse processo tendeu à otimização por convergência a uma melhor solução através da combinação de critérios geométricos e estruturais. A estrutura encontrada cumpriu os pré-requisitos por ter sua geometria desenvolvida através da aplicação do modelo performativo, além de ter tido sua eficiência estrutural comprovada ainda na etapa de estudo preliminar do projeto (Figura 16).

\section{Projeto para a montagem}

O processo de projeto com a mediação de tecnologias digitais paramétricas e algorítmicas permite que a forma seja definida pelos mais diversos parâmetros, sejam eles de geometria, produção ou montagem. No projeto da torre eólica a estética não reproduziu o resultado direto das tensões principais. Mas por que não fazer a estrutura seguindo exatamente o caminho das tensões? Na forma encontrada, a variação de rigidez dos perfis acontece de maneira geométrica por meio do uso de tubos concêntricos, sendo a variação de densidade na horizontal dada pelo próprio diâmetro da casca, nesse sentido um problema axissimétrico.

\section{Tabela 1 - Critérios de desempenho}

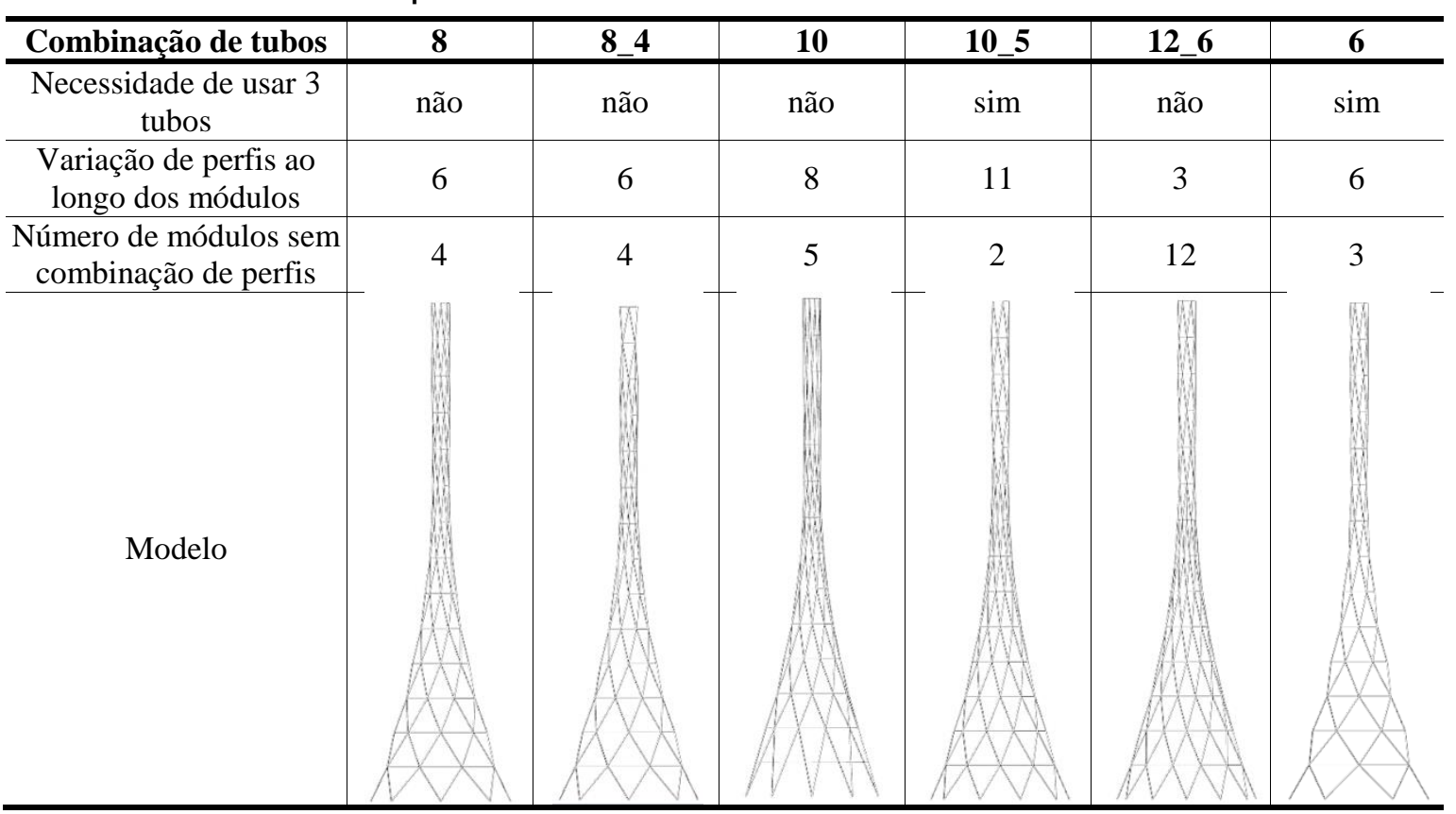

104 Borges, M. F.; Grilo, L. F.; Fakury, R. H. 
Figura 16 - Estrutura selecionada para desenvolvimento do projeto

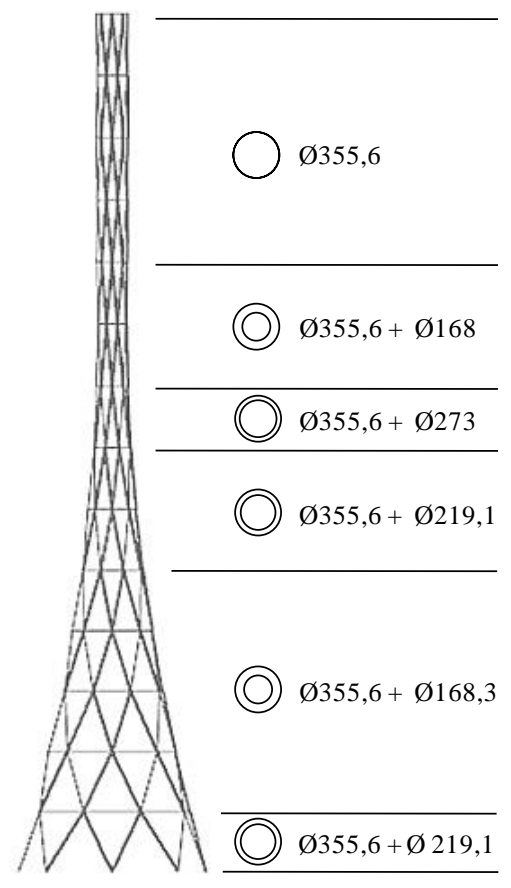

Após ser encontrada a forma ideal da modulação da torre para o fabricante, ainda na etapa de estudo preliminar, foram feitas algumas verificações das tensões no software SAP2000, em que foram testados os modelos com 8 e 6 barras por seção, já que foram aqueles que apresentaram os melhores resultados no processo desenvolvido para verificar o desempenho. A avaliação das formas foi feita utilizando-se o critério de peso e deslocamento da estrutura. Pode-se verificar na Figura 17 que nos modelos 1 e 3 o critério de peso não foi significativo para a decisão da forma.

Sendo assim, os parâmetros de modulação foram revistos com o intuito de verificar se as alterações nos ângulos e a diminuição do comprimento das barras gerariam uma diminuição significativa no peso da estrutura. Como pode ser verificado nos resultados dos modelos 2 e 4 , isso ocorreu, tendo diminuição de peso de respectivamente $16,75 \%$ e 12,89\%. Entretanto, com essas alterações de modulação, o número de ligações dobrou, aumentando custo de fabricação e montagem. Portanto, retornou-se ao critério de se evitar ao máximo o uso de tubos combinados, o que reforçou o melhor desempenho do modelo da torre com 8 nós. O modelo 1 foi a melhor opção, porque, além de não necessitar da combinação de perfis, permitiu que fossem utilizados perfis de mesmo diâmetro em toda a extensão da estrutura, variando apenas a espessura dos perfis em cada módulo.
Os resultados do modelo desenvolvido parametricamente foram validados pelo dimensionamento através do SAP 2000. Nesse programa utilizou-se a norma canadense CAN/CSA S16 (CANADIAN..., 2001), que emprega a mesma curva de resistência para barras comprimidas da norma brasileira NBR 16239 (ABNT, 2013), presente no programa. O modelo pode ser reutilizado para o projeto da torre sob diferentes condições, como local a ser implantado e material de fabricação. Dessa forma, pode-se dizer que foi construído um processo de customização em massa (Figura 18) para diferentes parques eólicos, podendo ser geradas, ainda no estudo preliminar, estruturas otimizadas sob o ponto de vista geométrico, de produção e de montagem. $\mathrm{O}$ modelo suporta alterações de parâmetros geométricos, de forças aplicadas e de seções transversais e resistência ao escoamento do aço dos tubos.

\section{Conclusão}

$\mathrm{O}$ projeto paramétrico e algorítmico tem potencial de viabilizar uma arquitetura em que o processo de criação do arquiteto está ligado ao gerenciamento de dados. O resultado final advém de um dos resultados possíveis dentro de uma infinidade de alternativas geradas, o que pode viabilizar uma abertura do processo de projeto para a colaboração de outros profissionais. Essa abertura diminui as fronteiras entre projeto e construção, separação esta muito fortalecida pelo projeto representativo. 
Figura 17 - Modelos analisados no SAP2000 - Perfis dos tubos verticais em cada módulo

$\frac{T C \frac{T C}{T} 55,6 \times 8,8}{\mathrm{TC} 355,6 \times 11} \frac{\mathrm{TC} 355,6 \times 14,2}{\mathrm{TC} 355,6 \times 17,5}$

Figura 18 - Customização em massa para diferentes parques eólicos
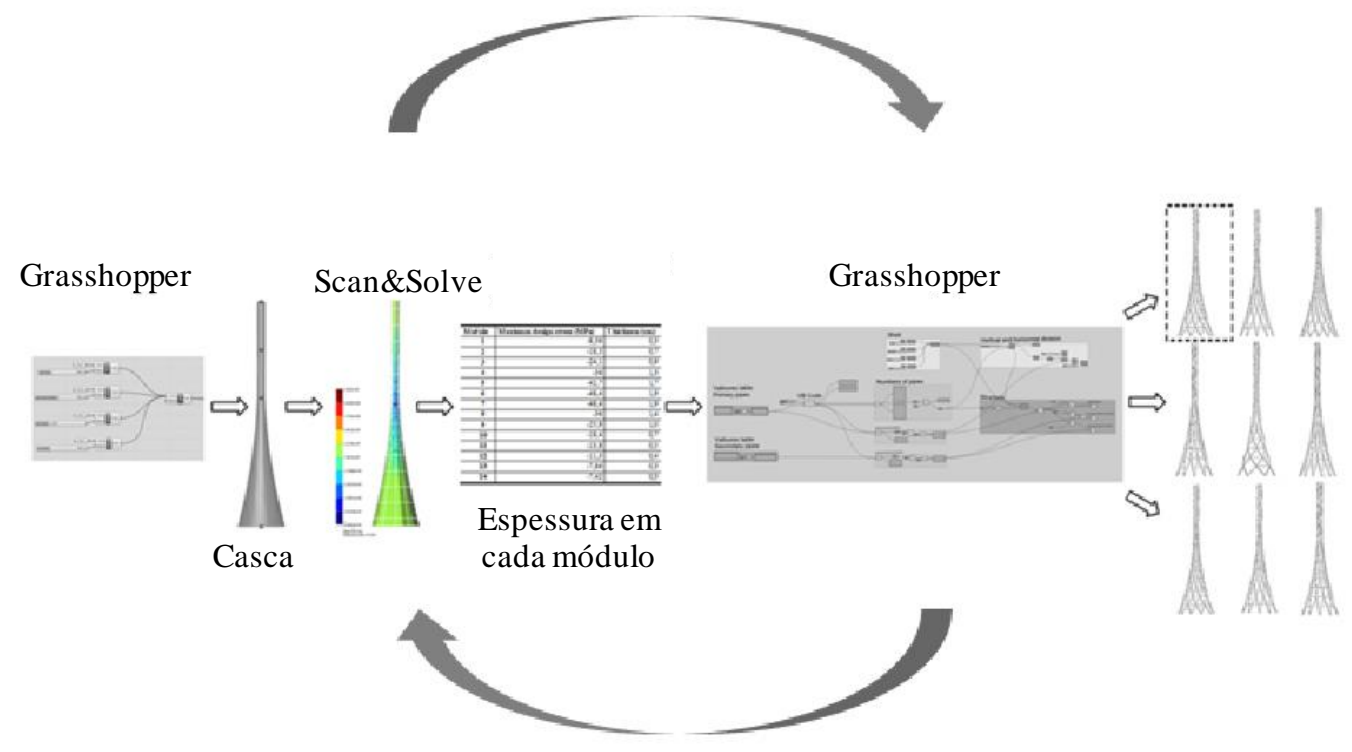

O processo digital cria um fluxo contínuo de projeto e produção. Sendo assim, com a ampliação de possibilidades atreladas ao projeto e com a abertura para a participação de outros profissionais na concepção da forma, cria-se a oportunidade de conceber projetos baseados em regras mais

racionais e menos intuitivas. Nesse contexto, os projetos baseados em desempenho passam a ser desenvolvidos, sendo prioridade a edificação responder a alguns parâmetros predefinidos e sendo a forma uma consequência dessas respostas. $\mathrm{O}$ projeto baseado em desempenho pode ser 
calcado apenas na otimização da forma concebida pelo arquiteto, ou pode abarcar sua própria geração. Em ambos os casos é fundamental que o processo seja colaborativo, com a participação do engenheiro de estruturas desde os estágios preliminares do projeto.

A metodologia apresenta um grande potencial para o desenvolvimento de estruturas complexas. Por meio de futuros trabalhos podem ser avaliadas as possibilidades de se aumentar a complexidade da análise estrutural, que pode passar a ser feita em teoria de segunda ordem e incluir o comportamento das ligações. Outras partes integrantes do projeto também poderiam ser incorporadas à análise, e, através de um objeto mais complexo, ser estudada como seria a correlação de mais de um componente ao se gerarem formas e avaliar o desempenho. Também poderia ser mais bem discutida a integração do projeto com a fabricação digital, além da integração do processo com fabricação e montagem. Ainda, os parâmetros de custos e tempo também poderiam ser incorporados aos processos de geração e fabricação da forma.

Embora não se possa afirmar que o projeto baseado em desempenho melhore a qualidade do projeto, observa-se que ele aumenta substancialmente as soluções e melhora a eficiência da estrutura ainda na fase de estudo preliminar. O processo colaborativo dilui as questões de autoria da forma, fazendo com que os conflitos entre autores (arquitetos) e avaliadores e materializadores (engenheiros) sejam diminuídos em prol de um melhor desempenho do projeto, através de processos investigativos e experimentais.

\section{Referências}

ANDREA, A. Condensed Version of Technical Report TR-04R0-2014-An: estimation of tower design loads for a generic $3 \mathrm{MW}$ wind turbine. Salvador, 2014.

ARCHDAILY. Estádio Olímpico de Munique. Frei Otto \& Gunther Behnisch. 25 fev.

2012. Disponível em:

<http://www.archdaily.com.br/br/01-

34759/estadio-olimpico-de-munique-frei-otto-egunther-behnisch>. Acesso em: 20 nov. 2014.

ASSOCIAÇÃO BRASILEIRA DE NORMAS TÉCNICAS. NBR 16239: projeto de estruturas de aço e de estruturas mistas de aço e concreto de edificações com perfis tubulares. Rio de Janeiro, 2013.
BURRY, J.; BURRY, M. The New Mathematics of Architecture. New York: Tames\&Hudson, 2010.

CANADIAN STANDARDS ASSOCIATION, CAN/CSA. S16 Limit States Design of Steel Structures. Ontario, 2001.

CELANI, G. Algorithmic Sustainable Design, Uma Visão Crítica do Projeto Generativo.

Vitruvius, São Paulo, v. 10, n. 116.03, 2011.

ENGSTRÖM, S. et al. Tall Towers For Large

Wind Turbines. Estocolmo: Elforsk, 2010.

GOMES FILHO, J. Gestalt do Objeto: sistema de leitura visual da forma. São Paulo: Escrituras, 2000.

GRILO, L. F. Estudo do Comportamento de Barras Compostas Comprimidas Formadas Por Perfis Tubulares Circulares Concêntricos Laminados de Aço. Belo Horizonte, 2015. Dissertação (Mestrado em Engenharia de Estruturas) - Escola de Engenharia, Universidade Federal de Minas Gerais, Belo Horizonte, 2015.

GROBMAN, Y. On Performalism: form and performance in digital architecture. In: GROBMAN, Y.; NEUMAN, E. (Eds.). The Various Dimensions of the Concept of "Performance" in Architecture. Routledge: Oxon, 2012.

KOLAREVIC, B. Performative Architecture Beyond Instrumentality. New York: Spon, 2005.

OXMAN, N. Material-Based Design

Computation. Massachusetts, 2010. Thesis (Ph.D.) - Massachusetts Institute of Technology, 2010.

OXMAN, R. Theory and Design in the First Digital Age. Design Studies, v. 27, p. 229-265, 2006.

OXMAN, R.; OXMAN, R. The New

Structuralism: design, engineering and architectural technologies. AD, v. 80, p. 15-23, 2010.

SCAN \& SOLVE. Scan\&Solve ${ }^{\text {TM }} 2014$ Plug-in

for Rhino. Version 2014.6.16.0. Madison, California: Intact Solutions, 2014. Disponível em: $<$ http://www.intactsolutions.com/site2014/download_sns_frontend.ph p>. Acesso em: 23 fev. 2014.

SHEA, K.; AISH, R.; GOURTOVAIA, M. Towards integrated Performance-Driven Generative Design Tools. Automation in Construction, v. 14, n. 2, p. 253-264, 2005. 
TERZIDIS, K. On Algorithmic Form. In: AHLQUIST, S.; MENGES, A. Computational Design Thinking. London: John Wiley \& Sons, 2011.

\author{
VALLOUREC TUBOS DO BRASIL. Tubos \\ Estruturais, Seção Circular, Quadrada e \\ Retangular. Catálogo. São Paulo, 2014.
}

\section{Marina Ferreira Borges}

Programa de Pós-Graduação em Arquitetura e Urbanismo, Escola de Arquitetura | Universidade Federal de Minas Gerais | Rua Paraíba, 697, Funcionários | CEP 30130-140 | Tel.: (31)3409-8829 | E-mail: marinafborges@gmail.com

\section{Lucas Figueiredo Grilo}

Departamento de Engenharia de Estruturas, Escola de Engenharia | Universidade Federal de Minas Gerais | Av. Pres. Antônio Carlos, 6627 Campus Pampulha, DEES, Bloco 1, Sala 4215, Pampulha | Belo Horizonte - MG - Brasil | CEP 31270-901 | Tel.: (31) 3409-1980 |

E-mail: lucasfgrilo@gmail.com

\section{Ricardo Hallal Fakury}

Departamento de Engenharia de Estruturas, Escola de Engenharia | Universidade Federal de Minas Gerais | Av. Pres. Antônio Carlos, 6627, Campus Pampulha, DEES, Bloco 1, Sala 4106, Pampulha | Belo Horizonte - MG - Brasil | CEP 31270-901 | Tel.: (31) 3409-1972 |

E-mail: fakury@dees.ufmg.br

\section{Revista Ambiente Construído}

Associação Nacional de Tecnologia do Ambiente Construído

Av. Osvaldo Aranha, $99-3^{\circ}$ andar, Centro

Porto Alegre - RS - Brasil

CEP $90035-190$

Telefone: +55 (51) 3308-4084

Fax: +55 (51) 3308-4054

www.seer.ufrgs.br/ambienteconstruido

E-mail: ambienteconstruido@ufrgs.br

108 Borges, M. F.; Grilo, L. F.; Fakury, R. H. 